\title{
On biaccessible points in Julia sets of polynomials
}

\author{
by
}

\author{
Anna Z d unik (Warszawa)
}

\begin{abstract}
Let $f$ be a polynomial of one complex variable so that its Julia set is connected. We show that the harmonic (Brolin) measure of the set of biaccessible points in $J$ is zero except for the case when $J$ is an interval.
\end{abstract}

1. Introduction. In the recent paper $[\mathrm{Za}] \mathrm{S}$. Zakeri proved that if the Julia set of a quadratic polynomial $f(z)=z^{2}+c$ is locally connected, then the set of biaccessible points has Brolin measure zero except when $f(z)=z^{2}-2$.

He also mentioned the conjecture (due to Hubbard and Lyubich) that this phenomenon should be general. I show how to verify it.

If the Julia set of a polynomial is connected, then $A_{\infty}$, the basin of attraction of $\infty$, is simply connected and we can take a Riemann map $R$ : $\mathbb{D} \rightarrow A_{\infty}$ so that 0 is mapped to $\infty$. If $J(f)$ is locally connected then $R$ extends continuously to the closed disc. In general, it is no longer the case, but for Lebesgue a.e. $\theta \in S^{1}$ the Riemann map has a nontangential limit at $\theta[\mathrm{Po}]$ and we can define the harmonic measure (evaluated at $\infty$ ) on $J(f)=\partial A_{\infty}$ as the image of the Lebesgue measure in $S^{1}$ under $R$.

The pull back of $f, g=R^{-1} \circ f \circ R$, is just $g(z)=z^{d}$ where $d$ is the degree of $f$.

Since the Lebesgue measure is invariant and ergodic under $g$, its image is invariant and ergodic under $f$. It is also the measure of maximal entropy. In the dynamical context it is often called the Brolin measure.

The Lebesgue measure on $S^{1}$ will be denoted by $m$, and the harmonic measure by $\omega$. An important property of $\omega$ is that its Jacobian is constant and equal to $d$.

2000 Mathematics Subject Classification: Primary 58F11.

Research supported by KBN grant 2 P03A 02512. 
We do not assume $J(f)$ to be locally connected. Let $P \subset S^{1}$ be the set of points $x$ so that the radial limit of $R$ exists at $x$. As we have noticed, $P$ is the set of full measure.

We will use the notation $R(x)$ for $x \in S^{1}$ even for the non-locally connected case, meaning by $R(x)$ the limit $R(r x)$ as $r \rightarrow 1$ (if it exists).

By construction, $\omega$-a.e. point $x \in J(f)$ is a landing point of some ray (i.e. there is $\theta$ so that $R(r \exp (i \theta)) \rightarrow x$ as $r \rightarrow 1)$. A point is called biaccessible if it is a landing point of two rays. By general topological reasons, only countably many points in the boundary of a simply connected domain admit three or more landing rays $[\mathrm{Po}]$.

We denote by $A$ the set of $\theta \in S^{1}$ so that the $\operatorname{limit} \lim _{r \rightarrow 1} R(r \theta)$ exists and there exists exactly one $\theta^{\prime} \in S^{1}, \theta^{\prime} \neq \theta$, such that $\lim _{r \rightarrow 1} R(r \theta)=$ $\lim _{r \rightarrow 1} R\left(r \theta^{\prime}\right)$. Observe that if $\theta \in A$ then $g(\theta) \in A$ unless $\theta$ lands in a critical point of $f$.

Thus, $J(f)$ is divided (up to a set of measure zero) into two $f$-invariant subsets: the biaccessible points and the set of points admitting one landing ray only.

By ergodicity, the measure of one of these sets is zero, the measure of the other is one.

We are going to prove

TheOREM. If $f$ is a polynomial so that the Julia set $J(f)$ is connected then the harmonic measure of the biaccessible points is zero except when $J(f)$ is an interval.

\section{Proof. Let}

$$
A_{\delta}=\{x \in A \text { : there exists } y \neq x \text { with } d(x, y)<\delta, R(x)=R(y)\}
$$

(here, $d$ is the distance in $S^{1}$ ). We start with a lemma:

Lemma 1. Assume that $A$ is dense. Then for every $\delta>0$ there exists an arc $L \subset S^{1}$ such that $\operatorname{diam}(L)<\delta$ and $A \cap L \subset A_{\delta}$.

Pr o o f. Let $R(x)=R(y)$ for some $x, y \in A$. These points divide $S^{1}$ into two $\operatorname{arcs} L_{1}$ and $L_{2}$.

If $R(w)=R(t)$ for some $w \in L_{1}$ and $t$, then both $w$ and $t$ belong to the same arc $L_{1}$ (since rays cannot intersect). Thus, we have a shorter arc $L_{3} \subset L_{1}$ with endpoints $w$ and $t$ such that if $x \in L_{3}$ and $R(x)=R(y)$ then $y \in L_{3}$.

By density of $A$ we could choose $w$ to lie "almost" in the middle of the $\operatorname{arc} L_{1}$. Then $L_{3}$ is (in the worst case) almost twice shorter than $L_{1}$. We continue by induction. 
There are certainly many ways to deduce easily the statement of the Theorem for expanding polynomials. (See [Ja] for the much stronger statement that in this case the set $A$ is countable.)

However, I give the proof for expanding maps in Propositions 1 and 2 below, in order to help the reader understand the general case.

Recall that the map $f$ is called expanding (on its Julia set) if there exists an integer $m$ such that $\left|\left(f^{m}\right)^{\prime}\right|>1$ in some neighbourhood of $J(f)$. A classical theorem says that $f$ is expanding iff the closure of the orbit of every critical point is disjoint from the Julia set.

If $J(f)$ is connected and $f_{\mid J(f)}$ is expanding then $J(f)$ is locally connected ([DH], Sec. 3), thus the Riemann map $R$ extends continuously to the closed disc.

Proposition 1. Let $f$ be a polynomial with connected Julia set so that $f_{\mid J(f)}$ is expanding. Then there exists $\delta>0$ such that for every arc $L \subset S^{1}$ of length $<\delta$ the map $R_{\mid L}$ is a.e. one-to-one (with respect to the Lebesgue measure).

Proof. We shall prove that $m\left(A_{\delta}\right)=0$ for small $\delta$.

First, notice that $f$ is a local homeomorphism in the neighbourhood of $J(f)$, i.e. there exists $\varepsilon>0$ such that $f$ is one-to-one in every ball with diameter $\varepsilon$. Since $R$ is continuous, there exists $\delta_{0}>0$ such that if $d(x, y)<\delta_{0}$ then $d(R(x), R(y))<\varepsilon$.

Now, since $R$ is a.e. at most two-to-one, there exists $\delta<\delta_{0}$ such that $m\left(A_{\delta}\right)<1$. Indeed, $\bigcap_{\delta} A_{\delta}=\emptyset$ and $A_{\delta_{1}} \subset A_{\delta_{2}}$ if $\delta_{1} \leq \delta_{2}$, thus $\lim _{\delta \rightarrow 0} m\left(A_{\delta}\right)=0$.

We check that $A_{\delta}$ is $g$-invariant, $g^{-1}\left(A_{\delta}\right) \subset A_{\delta}$. Let $x \in A_{\delta}$. There exists $y$ with $d(x, y)<\delta$ such that $R(x)=R(y)$. Let $x_{1}=g_{\eta}^{-1}(x)$ for some branch of $g^{-1}$. Let $y_{1}=g_{\eta}^{-1}(y)$. We have

$$
f\left(R\left(x_{1}\right)\right)=R\left(g\left(x_{1}\right)\right)=R\left(g\left(y_{1}\right)\right)=f\left(R\left(y_{1}\right)\right) .
$$

By our choice of $\varepsilon$ and $\delta$ this implies $R\left(y_{1}\right)=R\left(x_{1}\right)$. It follows that $g^{-1}\left(A_{\delta}\right)$ $\subset A_{\delta}$, thus $m\left(A_{\delta}\right)=0$ by ergodicity of $g$.

Fix $\delta$ as in Proposition 1.

Proposition 2. If $f_{\mid J}$ is expanding, then $m(A)=0$.

Proof. By Lemma 1 there exists an arc $L$ such that $A \cap L \subset A_{\delta}$. Using Proposition 1 we get $m(A \cap L)=0$, thus $m(A)<1$ and, by ergodicity, $m(A)=0$.

Now, we pass to the general case. 
Definition. A point $a \in S^{1}$ is called an end point if there exist sequences $a_{n}, b_{n} \in A$ such that

$$
a_{n} \rightarrow a, \quad b_{n} \rightarrow a, \quad a \in\left(a_{n}, b_{n}\right)
$$

(where $\left(a_{n}, b_{n}\right)$ is the shorter arc joining $a_{n}$ and $b_{n}$ in $S^{1}$ ) and $R\left(a_{n}\right)=$ $R\left(b_{n}\right)$.

Notice that if $a$ is an end point and $\theta \in\left(a_{n}, b_{n}\right) \cap A$, then the corresponding $\theta^{\prime}$ is in $A \cap\left(a_{n}, b_{n}\right)$ as well, since the rays cannot intersect.

Notation. Consider the Jordan curve $J_{n}$ defined as the union of three arcs: $R\left(r a_{n}\right), r \geq 1 / 2, R\left(r b_{n}\right), r \geq 1 / 2$, and the level line of Green's function: $R\left(\frac{1}{2} \varphi\right), \varphi \in\left(a_{n}, b_{n}\right)$.

Denote by $V_{n}$ the simply connected domain in $\overline{\mathbb{C}}$ bounded by the union of two arcs: $R\left(r a_{n}\right)$ and $R\left(r b_{n}\right)$, and containing the level line $R\left(\frac{1}{2} \varphi\right), \varphi \in$ $\left(a_{n}, b_{n}\right)$.

Denote by $O_{n}$ the simply connected domain bounded by $J_{n}$ and not containing $\infty$.

Lemma 2. If $A$ is dense and $J(f)$ is not an interval then there are infinitely many end points.

Proof. First notice that (as indicated in the proof of Lemma 1) if $R(a)=$ $R(b)$ then there are at least two ends, one in each arc bounded by $a, b$ in $S^{1}$.

Assume that there are more than two ends.

We build Thurston's lamination. For $\theta \in A$ we join it to the corresponding $\theta^{\prime}$ with a hyperbolic geodesic. Let $L$ be the union of such geodesics, and let $\bar{L}$ be the closure of $L$.

Now, it is easy to observe that if there are more than two ends then there exists a gap (in Thurston's terminology), i.e. the set $D \backslash \bar{L}$ is nonempty. A gap is a component of $D \backslash \bar{L}$. But then (by invariance of lamination) there are infinitely many gaps and, moreover, gaps are dense in $D$ ([Th], Prop. 10.1).

This produces infinitely many ends.

Thus, we are left with the case of two ends $a, b$. Since the image (under $g$ ) of an end is an end, we have

$$
g(\{a, b\}) \subset\{a, b\} .
$$

Taking $g^{2}$ instead of $g$, we may assume that $g(a)=a$.

Since $a$ is a fixed point of $g$, there exists a radial limit of $R$ along the ray $r \cdot a, R(a)=x$. This is a well known fact (due to Fatou); the reader may find the proof in $[\mathrm{P}]$.

Let $g_{\nu}^{-1}$ be the branch of $g^{-1}$ in the cone

$$
\left\{r \phi: 0<r<1, \phi \in\left(a_{m}, b_{m}\right)\right\}
$$


such that $g_{\nu}^{-1}(a)=a$. (Here, $a_{m}, b_{m}$ are as in the definition of the end point, sufficiently close to $a$.) Then there is a branch $f_{\nu}^{-1}$ in $V_{m}$ such that $f_{\nu}^{-1}=R^{-1} \circ g_{\nu}^{-1} \circ R$.

(Otherwise, the component of $f^{-1}\left(V_{m}\right)$ containing $x$ would be a domain bounded by a union of more than two rays,

$$
R\left(r \theta_{1}\right), R\left(r \theta_{1}^{\prime}\right), \ldots, R\left(r \theta_{k}\right), R\left(\theta_{k}^{\prime}\right),
$$

$k>1, r \leq 1, R\left(\theta_{i}\right)=R\left(\theta_{i}^{\prime}\right)$. Notice that each pair $\theta_{i}, \theta_{i}^{\prime}$ produces at least one end different from $a$. For different pairs we get different ends. This gives at least three ends.)

Observe that $f_{\nu}^{-1}\left(V_{m}\right) \subset V_{m}$ since $g_{\nu}^{-1}\left(\left(a_{m}, b_{m}\right)\right) \subset\left(a_{m}, b_{m}\right)$. Also, we have $\operatorname{cl}\left(\left(f_{\nu}^{-1}\left(O_{m}\right)\right) \subset O_{m}\right.$. This gives $\operatorname{diam}\left(\left(f_{\nu}^{-1}\right)^{n}\left(O_{m}\right)\right) \rightarrow 0$. This implies that

$$
\bigcap_{k}\left(O_{k} \cap J\right) \subset \bigcap_{n}\left(f_{\nu}^{-1}\right)^{n}\left(O_{m}\right)=\{x\} .
$$

Now, consider the second end point $b$. Then either $b$ is another fixed point of $g$ and the same reasoning applies, or $g(b)=a$.

Consider the domains $O_{k}^{\prime}, V_{k}^{\prime}$ build in the same way as in the previous case, but corresponding to $b$.

Then $f\left(O_{k}^{\prime}\right)$ can be taken as the family $O_{k}$ corresponding to $a$ and we have

$$
f\left(\bigcap_{k}\left(O_{k}^{\prime} \cap J\right)\right) \subset \bigcap_{k} f\left(O_{k}^{\prime} \cap J\right) \subset \bigcap_{k} O_{k} \cap J=\{x\} .
$$

We conclude that $\bigcap_{k}\left(O_{k}^{\prime} \cap J\right)$ is a point, which we denote by $y$.

Now, if $p \in J, p \neq x, y$, is a critical value, take a domain $D$ in $\overline{\mathbb{C}}$ bounded by two pairs of rays, one pair $a_{m}, b_{m}$ corresponding to the end $a$, the other one corresponding to the end $b$.

For $m$ large, $p$ is in $D$, by the above observation. If $c$ is a critical point such that $f(c)=p$, take a component of $f^{-1}(D)$ containing $c$. Its boundary is built of more than two pairs of rays, each of them produces an end. Again, there are more than two ends. It follows that $x, y$ are the only critical values in $J$.

Similarly, one can check that every preimage of $x$ or $y$ different from $x, y$ must be a critical point of degree two.

The only polynomials satisfying the above conditions are Chebyshev polynomials (up to sign) (see e.g. [Z]). The corresponding Julia set is an interval.

Under the assumption of Lemma 2 we fix a large integer $M$, an end point $a$ and the corresponding $a_{m}, b_{m}$ so that there are no critical values of $f$ up to order $M$ in $\operatorname{cl}\left(O_{m}\right)$.

To simplify notation, we denote this $O_{m}$ just by $O$. 
Now, we use a lemma which makes the situation similar to the expanding case.

LEMma 3. If $M$ is large enough, then there exists a countable family $\left\{U_{s}\right\}$ of topological discs so that $\operatorname{cl}\left(U_{s}\right) \subset O, \operatorname{cl}\left(U_{s}\right)$ are pairwise disjoint and

$$
\omega\left(\bigcup U_{s}\right)=\omega(O)
$$

such that each $U_{s}$ is the image of $O$ under an analytic branch of $f^{-n(s)}$ defined in $O$ (for some $n(s) \geq M)$.

Proof. If $f_{\mid J(f)}$ is expanding then the construction below is simply the first return map to $O$. Here, the construction of backward branches defining $U_{n}$ 's is also based on the first return technique, but it has to be done in the (Rokhlin) natural extension (the space of backward trajectories) after restricting to the set of suitable branches. (See e.g. [FKS] or [PUbook] for more details on natural extension.)

Define the space of trajectories by

$$
X=\left\{\widetilde{x}=\left(\ldots, x_{-1}, x_{0}, x_{1}, \ldots\right): x_{i} \in S^{1}, g\left(x_{i}\right)=x_{i+1}\right\} .
$$

The one-to-one map $X \rightarrow X$ is the left shift $\sigma$.

The measure $m$ can be lifted to a measure $\widetilde{m}$ which is $\sigma$-invariant and ergodic. It is defined on cylinders by

$$
\begin{aligned}
\widetilde{m}\left(X \cap \left(\ldots \times S^{1} \times S^{1} \times A_{-n} \times\right.\right. & \left.\left.\ldots \times A_{0} \times S^{1} \times \ldots\right)\right) \\
& =m\left(A_{0} \cap g^{-1}\left(A_{1}\right) \cap \ldots \cap g^{-n}\left(A_{n}\right)\right) .
\end{aligned}
$$

Let $\pi: X \rightarrow S^{1}$ be the natural projection defined as $\pi(\widetilde{x})=x_{0}$. Then $g \circ \pi=\pi \circ \sigma$ and $\pi_{*}(\widetilde{m})=m$.

(Actually, we do not need this general construction here, since $g$ is isomorphic to the one-sided Bernoulli shift $(1 / d, 1 / d, \ldots, 1 / d)$ and $X, \sigma$ is simply the full two-sided shift.)

We consider all backward branches of $g^{-n}$ defined in the neighbourhood of the arc $\left[a_{m}, b_{m}\right]$ in $\mathbb{C}$.

We call such a branch good if the map $R \circ g^{-n} \circ R^{-1}$ extends to some holomorphic branch of $f^{-n}$ defined in the whole $O$.

Lemma 4. If $M$ is large enough, then there exists a set $G \subset \pi^{-1}\left(\left[a_{m}, b_{m}\right]\right)$ such that $\widetilde{m}(G)>0, \pi(G)=\left[a_{m}, b_{m}\right]$ and if $\widetilde{x} \in G$ then $x_{-n}$ is the image of $x_{0}$ under a good branch of $g^{-n}$.

Pr o of. This is a simple version of a well known phenomenon; the idea comes from [FLM].

First, we consider all $\left(d^{M}\right)$ branches of $g^{-M}$ in the neighbourhood of $\left[a_{m}, b_{m}\right]$. By our choice of $M, m$ they are all good. Among these branches 
there are at most $d-1$ branches such that there is a critical value of $f$ in the image of $O$ under the corresponding branch of $f^{-M}$.

We exclude these wrong branches and continue (compose with branches of $g^{-1}$ ) with the remaining ones.

Thus, if $a_{n+M}$ is the number of good branches of $g^{-(n+M)}$, we have

$$
a_{n+M+1} \geq d a_{n+M}-(d-1)
$$

and so

$$
\frac{a_{n+M+1}}{d^{n+M+1}} \geq \frac{a_{n+M}}{d^{n+M}}-\frac{d-1}{d^{n+M}} .
$$

Thus, if $M$ has been chosen large enough, there is a constant $t>0$ such that

$$
\frac{a_{n+M}}{d^{n+M}}>t .
$$

The set $G$ is obtained by attaching to each point $x \in\left[a_{m}, b_{m}\right]$ the sequence of its preimages under good branches chosen as above.

REMARK. Actually, in this particular case of maximal entropy measure, already $M=2$ is sufficient. Lemma 4 (and, consequently, the whole theorem) can be proved in the similar way for a wider class of invariant measures (including Gibbs states for Hölder continuous functions defined in $S^{1}$ ). This requires slightly more delicate estimates and large $M$.

Let $\sigma_{G}: G \rightarrow G$ be the first return map to $G$ under $\sigma^{-1}$. For every $\widetilde{x} \in G$ let $g_{\eta}^{-n}$ be the corresponding sequence of backward branches, set

$$
x_{-n}=g_{\eta}^{-n}\left(x_{0}\right)
$$

and let $f_{\eta}^{-n}$ be the corresponding sequence of backward branches in $O$.

Now, $\pi\left(\sigma_{G}(\widetilde{x})\right)=g_{\eta}^{-n}\left(x_{0}\right)$ for some $n$. Let $O_{\widetilde{x}} \subset O$ be the image of $O$ under the corresponding branch of $f^{-n}$.

For two different $\widetilde{x}$ 's the domains $O_{\widetilde{x}}$ are either disjoint or one is contained in the other. (Recall that $O$ is bounded by external rays landing at the same point.)

Suppose that one domain is contained in another one, say $f_{\eta}^{-(n+k)}(O) \subset$ $f_{\nu}^{-n}(O)$. Then going $k$ steps back along the first branch we fall into the domain $O$ of the second branch, going $n$ steps back we are in $G$ (so all further backward branches are defined in the whole $O$ ). But this means that already after $k$ steps back along the first branch we were in $G$, so this was already the first return to $G$.

It follows that the domains are either disjoint or coincide.

Also, $\bigcup_{\widetilde{x}} O_{\widetilde{x}}$ cover the domain $O$ up to a set of measure zero (since $\sigma_{G}: G \rightarrow G$ is a measure preserving isomorphism) ([FKS], Ch. 1.5).

This is the cover we are looking for.

This ends the proof of Lemma 3. 
So we have a new map $F$ defined $m$-a.e. in $O$ by

$$
F(z)=f^{n(s)}(z)
$$

for $z \in U_{s}$. Let

$$
X_{\infty}=\bigcap_{k=0}^{\infty} F^{-k}\left(\bigcup U_{s}\right) .
$$

We have the following easy

Proposition 3. $F: X_{\infty} \rightarrow X_{\infty}$ preserves the measure $\omega_{\mid X_{\infty}}$.

Proof. Since the Jacobian of $\omega$ equals $d$, the degree of $f$, we have $\omega\left(U_{s}\right)=\omega(O) / d^{n(s)}$. Thus, $\sum_{s} 1 / d^{n(s)}=1$. For any measurable subset $G$ of $X_{\infty}$ we have

$$
\omega\left(F^{-1}(G)\right)=\sum_{s} \frac{1}{d^{n(s)}} \omega(G)=\omega(G) .
$$

REMARK. Notice that the construction which replaces the original map by a piecewise expanding dynamics appeared in [Ma], then it was extended to a larger class of measures in [DU] (see also [DMNU]). All these papers deal with a more general dynamics of an arbitrary rational map. On the other hand, the statement of Lemma 3 does not follow directly from those results.

Proposition 4. For small $\delta$ we have $m\left(A_{\delta} \cap\left(a_{m}, b_{m}\right)\right)=0$.

Proof. Let $B_{\delta}=R\left(A_{\delta} \cap\left(a_{m}, b_{m}\right)\right)$. Then $B_{\delta} \subset O$. We check that $B_{\delta}$ is $F$-invariant, even more: $F^{-1}\left(B_{\delta}\right) \subset B_{\delta / d}$.

Indeed, let $x \in B_{\delta}$. Then $x$ is a landing point of two rays $R(r \theta), R\left(r \theta^{\prime}\right)$, $\theta, \theta^{\prime} \in\left(a_{m}, b_{m}\right)$. Let $y \in F^{-1}(\{x\})$. Then $y$ is the image of $x$ under a univalent branch of $f_{\nu}^{-s}$ defined in $O$ and the formula

$$
g_{\nu}^{-s}(w)=R^{-1} \circ f_{\nu}^{-s} \circ R(w)
$$

defines a holomorphic branch of $g_{\nu}^{-s}$ in

$$
\left\{w=r \varphi: 1>r \geq 1 / 2, \varphi \in\left(a_{m}, b_{m}\right)\right\} .
$$

Thus, for $\eta=g_{\nu}^{-s}(\theta), \eta^{\prime}=g_{\nu}^{-s}\left(\theta^{\prime}\right)$,

$$
R\left(g_{\nu}^{-s}(r \theta)\right)=f_{\nu}^{-s}(R(r \theta)) \rightarrow f_{\nu}^{-s}(x)=y .
$$

But $g_{\nu}^{-s}(r \theta)=r^{-d^{s}} \eta$, so $R(r \eta) \rightarrow y$ as $r \rightarrow 1$. Similarly, $R\left(r \eta^{\prime}\right) \rightarrow y$. Since $\eta$ and $\eta^{\prime}$ are obtained with the use of the same branch of $g^{-s}, d\left(\eta, \eta^{\prime}\right)<\delta / d$. So $y \in B_{\delta / d}$.

Thus, we have verified that $F^{-1}\left(B_{\delta}\right) \subset B_{\delta / d}$.

Now, since $\omega\left(B_{\delta}\right) \rightarrow 0$ as $\delta \rightarrow 0$ (because $\omega\left(\bigcap B_{\delta}\right)=0$ ), we get 


$$
\omega\left(B_{\delta}\right)=\omega\left(F^{-1}\left(B_{\delta}\right)\right) \leq \omega\left(B_{\delta / d}\right) .
$$

Using the same reasoning by induction we get

$$
\omega\left(B_{\delta}\right) \leq \omega\left(B_{\delta / d^{k}}\right) \rightarrow 0
$$

as $k \rightarrow \infty$. Since $B_{\delta}=R\left(A_{\delta} \cap\left(a_{m}, b_{m}\right)\right)$, we conclude that $m\left(A_{\delta} \cap\left(a_{m}, b_{m}\right)\right)$ $=0$.

Now, recall that we have started with the endpoint $a \in\left(a_{m}, b_{m}\right)$. For $n$ large we have

$$
\left(a_{n}, b_{n}\right) \subset\left(a_{m}, b_{m}\right)
$$

and $d\left(a_{n}, b_{n}\right)<\delta$ with $\delta$ chosen as in Proposition 4. Thus $A \cap\left(a_{n}, b_{n}\right) \subset$ $A_{\delta} \cap\left(a_{m}, b_{m}\right)$. This implies $m\left(A \cap\left(a_{n}, b_{n}\right)\right)=0$, since $m\left(A_{\delta} \cap\left(a_{m}, b_{m}\right)\right)=0$.

But $m(A)=0$ or $m(A)=1$; the second possibility has been excluded, thus $m(A)=0$.

This ends the proof of the Theorem.

Added in proof (January 2000). Recently I received a preprint On support of $d y$ namical laminations and biaccessible points in polynomial Julia sets by Stanislav Smirnov. It contains an independent proof of the result stated here.

Smirnov's approach is based on Beurling's estimates on harmonic measure; then he reduces the problem to my theorem on rigidity of the maximal measure $([\mathrm{Z}])$.

\section{References}

[DMNU] M. Denker, R. D. Mauldin, Z. Nitecki and M. Urbański, Conformal measures for rational functions revisited, Fund. Math. 157 (1998), 161-173.

[DU] M. Denker and M. Urbański, Ergodic theory of equillibrium states for rational maps, Nonlinearity 4 (1991), 103-134.

[DH] A. Douady et J. Hubbard, Etude dynamique des polynômes complexes (première partie), Publ. Math. d'Orsay, 84-02.

[FKS] S. Fomin, I. Kornfeld and Ya. Sinai, Ergodic Theory, Springer, Berlin, 1982.

[FLM] A. Freire, A. Lopes and R. Mañé, An invariant measure for rational maps, Bol. Soc. Brasil. Mat. 14 (1983), 45-62.

[Ja] M. Jakobson, On the classification of polynomial endomorphisms of the plane, Mat. Sb. 80 (1969), 365-387 (in Russian).

[Ma] R. Mañé, On the Bernoulli property for rational maps, Ergodic Theory Dynam. Systems 5 (1985), 71-88.

[Po] C. Pommerenke, Boundary Behaviour of Conformal Maps, Springer, Berlin, 1992.

[P] F. Przytycki, Remarks on simple connectedness of basins of sinks for iterations of rational maps, in: Banach Center Publ. 23, PWN, 1989, 229-235.

[PUbook] F. Przytycki and M. Urbański, Fractals in the Plane-Ergodic Theory Methods, to appear; preliminary version at www.math.unt.edu/urbanski.

[Th] W. Thurston, On the dynamics of iterated rational maps, preprint. 
[Za] S. Zakeri, Biaccessibility in quadratic Julia sets, I: the locally connected case, preprint SUNY, Stony Brook, 1998.

[Z] A. Zdunik, Parabolic orbifolds and the dimension of the maximal measure for rational maps, Invent. Math. 99 (1990), 627-649.

Institute of Mathematics

Warsaw University

Banacha 2

02-097 Warszawa, Poland

E-mail: aniazd@mimuw.edu.pl

Received 25 May 1999;

in revised form 20 September 1999 